\title{
Morphometry of Sardine Otoliths Using Digital Image Processing
}

\author{
Kandeel Fatima \\ Department of Biomedical Engineering, \\ Mehran University of Engineering \& Technology, \\ Jamshoro, Pakistan. \\ kandeel_fatima@hotmail.com
}

\begin{abstract}
Otoliths of fish have since quite a while ago assumed an essential part in economical fisheries administration. Stock evaluation models at present utilized depend on species particular age profiles got from the occasional examples of development denotes that otoliths show. An ongoing expansion to shape demonstrating methods to figure out which performs best. An investigation is done into Impact of segmentation methods are considered to decide whether automated techniques is used for boundary extraction are more meaningful and whether built classifiers can be used at various establishments. The study of otolith and qualified researches reveals the exceptional development based on the life, stock structure, age, history, growth, migration, mortality, discoveries in marine biology and reveals the importance of fisheries management This research analysis characterized by the many techniques of image processing and the methods related with studies of otolith microstructure. The entire progression of proceedings from sample images collection to data processing is done comprehensively to make otolith analysis much advance and improved.
\end{abstract}

Keywords-Otolith, Thresholding, Region growing, Image processing.

\section{INTRODUCTION}

Otolith is a white small flake like substance that is situated in the inner ears of fish. They nurture constantly due to growth process. When the accumulation development is effected by environmental parameters and physiological conditions then otoliths can be used as fisheries biological records. This information extracted by otolith conditions can be useful for the studies of fish stock and ecology. By the collection and assessment of data occurrence, age, stock and habitat could be estimated. Decoding this data to estimate and interpret the otoliths is quite challenging because of the circumstances influencing the accumulation frequently. An assortment of analysis on the basis of both properties chemical and physical properties of otolith can be detected by processing of otolith images or Visual examination of images of developed otolith by segment, being evaluated by computer vision techniques applying image processing algorithms to get better quantitative and automatic assessment. These methods would let to entirely deem the images of otolith by mean of quantitative analysis. Otolith images formed with lines and marks on its structures similar to the one found in tree trunk. Numerous researchers have worked on such images to take out these lines boundaries and curves, using multiple methods and active contours although got better outcomes on the class considered as simple to estimate, but on extra complicated images the suggestions ended were limited. The processing and visualization of otolith of sardine fish images that are concentric structures in otolith images that were complex to interpret, done finely by the computer vision using Otsu method with manual and automatic thresholding values . The assessment of computer vision image processing is pioneering to an innovative approach to the extraction of otolith image data by getting fine lines 
boundaries and curves also the revival of the successive otolith's shapes throughout its ecology and growth. This is an easy and novel approach as framework for digital images, to be applied to sardine fish otolith images data set. The outcomes of proposed approach are given in the images, this research also providing a novelty to the image processing techniques that could be useful for fisheries management and further ecological studies. The digital imaging tool to enhance otolith microstructure for estimating age in days in juvenile and adult fish. This research is an analysis of fish otolith a microstructure to estimate the age of fish. This research is based upon the simulations and new software that is helpful for marine biologists to read microstructure of otolith of fishes very efficiently. The methodology comprises of three steps:

A) The blind image registration technique fast normalized cross correlation a single gray scale digital image combining images of different parts by using of the same fish otolith

B) The adaptive histogram equalization technique has been used for age approximation determinations in which the growth rings of the image are improved for this estimation

C) The microstructures are easily identifiable with help of a semiautomatic interactive tool that draws a simple multilateral chain along the points of interest to be marked, and the data will be hold back automatically.

This analysis of microstructure of fish otolith fish consumes lot of time and it is repetitive. The limitation of software is to analyze images proficient of both overlaying images automatically and recording a high number of daily growths in counting and measuring increments in large otolith [1]. Preliminary data on the study of otolith morphology of five pelagic fish species from the Adriatic Sea (Croatia), this paper is based on comparative taxonomy. To analyze five measurements of Otolith from pelagic species during the growth were examined by taking samples from quadratic sea and digitize the image using couple camera and then interface to an OLYMPUS binocular microscope and computer. To calculated the description of the dimensional shape of form factors, roundness, aspect ratio, and maximum length of otolith and the weight of otolith by using shape analysis of morphometric characters. The future work is that to identify the measurement of geometric shape planning to verify the role of otolith shape fluctuation [2] Otolith research and application: current directions in innovation and implementation. Gavin A. Begg. (2005) assembled on comparative investigation, reviewed on 11 to $16^{\text {th }}$ of July (2004) at Townville, Queensland, Australia was held on the third international symposium based on research and application of otolith. At the symposium addressed eight issues for specific fishes, sharks and invertebrate's themes such as structure and function, age, growth factors, chemical composition, statistics, stock calculation of fisheries management, aging created quality control and technological progresses. The paper based on eight themes accessible in paper in the special issues. At the symposium they provide information detailed about life history of otolith and related biological population and delivered data of fisheries stock to determining the sustainability [3].Assessment of the morphometry of saccularotoliths as a tool to identify triplefin species (Tripterygiidae). To determine the variability of cluster analysis and discriminated analysis between species to find the identification of species by using an algorithm PCA (principal component analysis) to performed the characterization by means of morphometric of otoliths. This evidence will be a beneficial tool for paleontological, taxonomic and trophic ecology consideration [4].

\section{METHODOLOGY}

Datasets were obtained with the information of individual samples. The different number of data transformations are used to transform the boundaries are extracted and compared by modified methods across datasets to find which method returns best results on specified a number of factors. Comprising the age of otolith samples, the algorithms used in the research by evaluated mean opinion score (MOS) combined with associated testing diagrams to determine the factors impacting on the classification of results and which choice of method returns the better results. Results are compared across Boundary segmentation method and learning algorithm. 


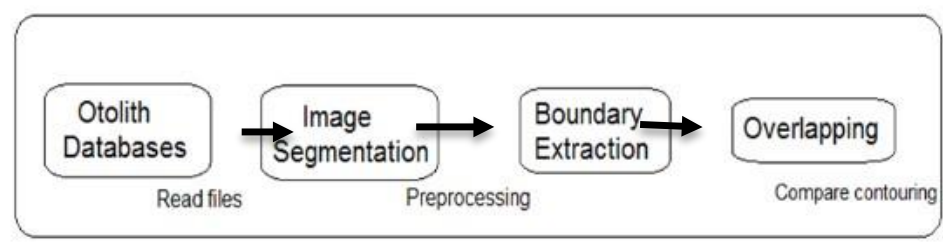

Fig. 1. Flow Diagram of Image Processing Segmentation Was Completely Carried Out In MATLAB Software

A) Working algorithm using MATLAB for morphological reconstruction of sardine otolith

B) Working algorithm using MATLAB for segmentation using thresholding of sardine otolith

C) Working algorithm using MATLAB for segmentation using region growing of sardine otolith

Segmentation can be applied to original images as well as it can be used after the applying filters or transformations on the basic approaches of segmentation include Thresholding, Edge-based methods and Region-based methods.

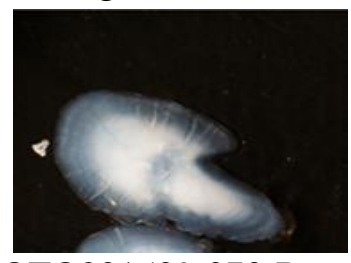

OTO201509-073-R

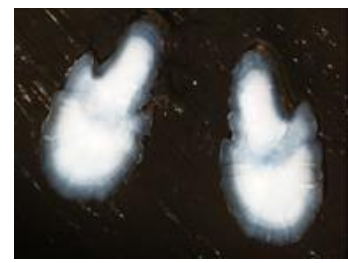

OTO201412-036 R-L

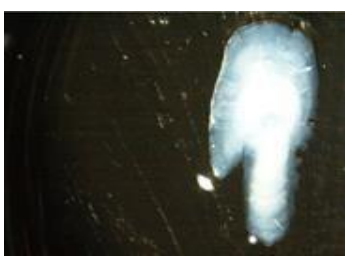

OTO201412-043-L

Fig. 2. Image Acquisition Setup (data sets)

Species studied in this Research the left and right sagittal otoliths may be used for study. This information provide particulars concerning a project developed between the Image Processing research groups (TIC128 of University of Malaga). The study has been organized by two phases.

D) Samples selection of sardine otolith called biological part which provided by IEO (Instituto Español de Oceanografía).

E) Images processing algorithms development called Engineering part.

\section{IMAGE PROCESSING ALGORITHIMS}

This research study is focused upon two methods which are applied on MATLAB for processing of otolith images of sardine fish. A basic approach of segmentation includes both Thresholding and Region-based methods.

\section{A. Thresholding Based Segmentation Using MATLAB}

In this research we performed different kind of image processing to get better results. The poor quality of the images due to way of obtaining was identified as: 
ISSN: 2446-2918 DOI: 10.21058/gjecs.2019.32007

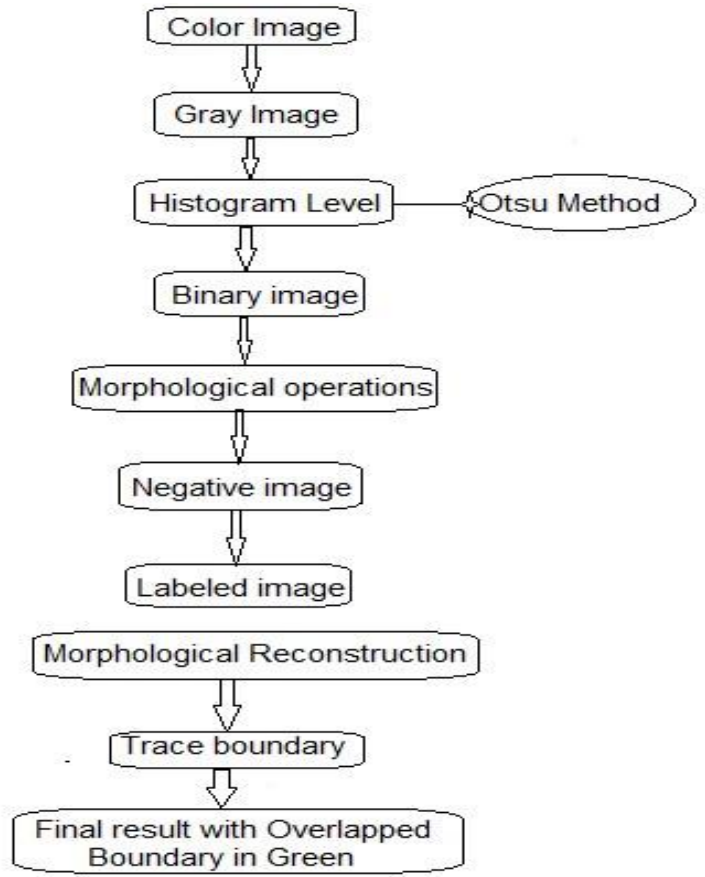

Fig. 3. Block diagram of steps of image processing by Otsu Technique

First data set of colored images is taken then these images converted to gray and then histogram level was applied using otsu method to binarise image. After binarization, morphological operation was performed on the binarised images. Bound extraction of is done by morphological reconstruction that shows overlapped boundaries as result because there is possibility to have two or three objects present in pictures needed to remove and to have a judgment that will make a possible outcome with a comparison of input and output image.

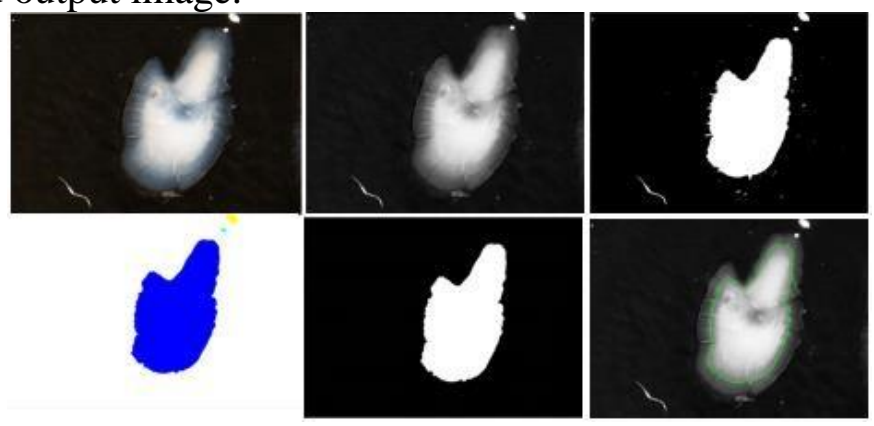

Fig. 4. Comparison between input and output images by Segmentation of Otsu Technique

The overlapping of gray image and the reconstructed image is done to obtain the outer contour in green color. As shown in above figure4, the accuracy of the image is not good because the contour is not well defined. Image processing of the sardine's otolith is done after running the algorithm which is obtaining the contour of the initial and final image more accurately. The first error is found in outer contouring, is that the resulting image is shrinked (diminished) too much because of performing morphological reconstruction to the image to get output, that is not in a good contouring.[5]The second error is found which seems in the image because of shadows created by the light reflection in the resin wherever the otoliths were placed. It means this algorithm was not good to get outcome to measure the shape. For this purpose, main thing is changing "threshold level " which is an improved code as it can be seen below. Steps are followed:

A) Using MATLAB read and display the image.

B) Applying threshold level for histogram related to background

C) Produce a structure element of disk shape to conserve the circular nature of object Perform a morphological operation on the image 
D) Overlapped image with output result

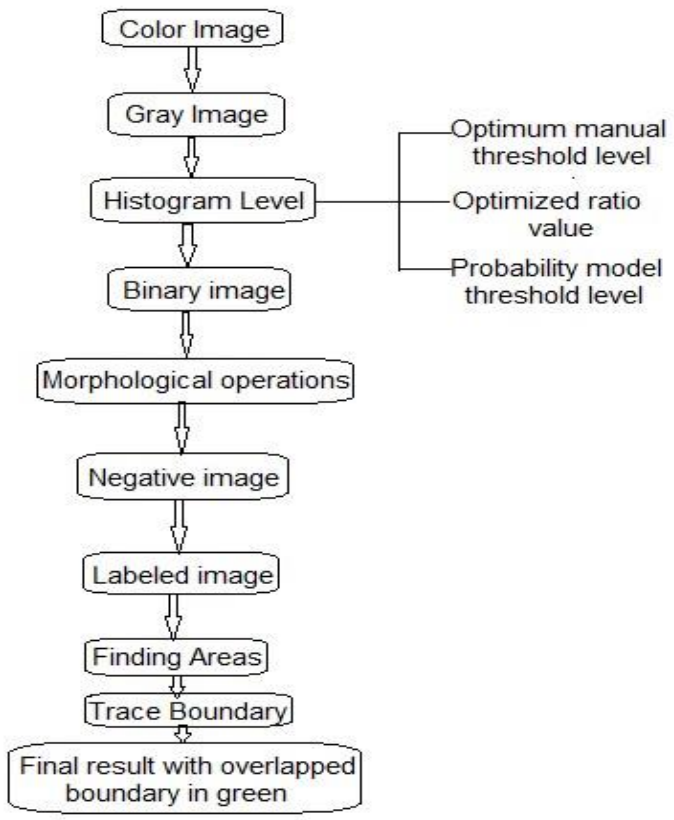

Fig. 5. Segmentation Based Thresholding Algorithm using MATLAB

By applying morphology image processing the gray image is converted into binary in order to generate the processing in an easier way by method in which the threshold is nominated to select by using gray thresh function this method is called Otsu's method. Gray thresh has a value of threshold between 0 and 1 . Adjusting the initial threshold by removing the histogram or manually modifying the level of threshold. In a previous part of algorithm, the result is not accurate with overlapping, now designing next algorithm to perform a better contouring by removing reconstruction and to put the level of threshold from histogram values in the gray image to change into the binary image. The idea is to obtain a good threshold value [6] [12]. Selecting an optimum manual threshold value is the most important step in image thresholding algorithms. The most important step here is to choose the best value for the threshold to get the best segmentation. The key reason is to choose "threshold level of histogram" associated to background because of its inhabitant properties and easiness of performance, image thresholding admires a central state in presentation of image segmentation.
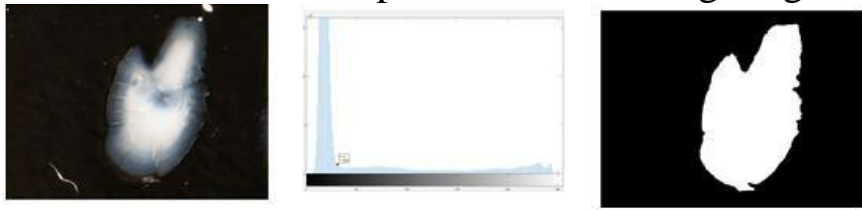

Fig. 6. a) Input image b) Threshold level c) Output image

\section{COMPARISON OF ALGORITHMS}

Comparison is carried out between overlapped images between algorithm 1 (Otsu) and algorithm 2 (optimum manual threshold):

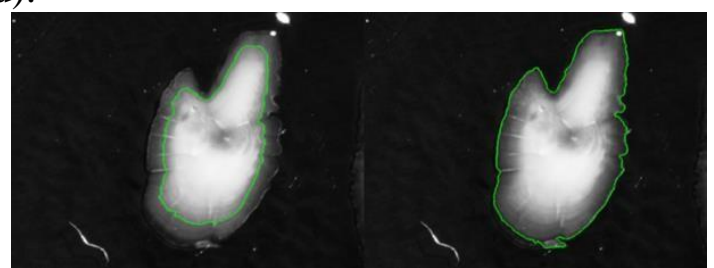

Fig. 7. Otolith image of comparison between algorithm 1 and 2

Most common error was found in first algorithm obtaining contour in image is related to the threshold level which shows deformed the shape of the otolith with overlapping. When we choose 
the level doesn't relate to background to get a poor result. Second image shows better result than previous because in which we choose manually selection of threshold level so, intensities of histogram expose the extraordinary contrast and sharpness that is occupied by optimum thresholding level, it demonstrations taking level at the starting point show good overlapping because of low contrast intensity in an image of data set [7].

Table 1. Comparison of methods

\begin{tabular}{|l|c|c|}
\hline $\begin{array}{l}\text { Methods } \\
\text { comparisons }\end{array}$ & Otsu Method & $\begin{array}{c}\text { Optimum manual } \\
\text { threshold level }\end{array}$ \\
\hline Advantage & $\checkmark$ Automatic & $\checkmark$ Good results \\
& $\checkmark$ Fast & \\
& $\checkmark$ Less time & \\
\hline Disadvantage & $\checkmark$ Bad result & $\checkmark$ Non Automatic \\
& & $\checkmark$ Time consuming \\
\hline
\end{tabular}

We need to raise image enhancement with selection of background threshold level based on image for further improving image processing techniques. In figure 8 done with first two steps, and in below further two steps are shown. We discus over here ways of choosing the threshold value automatically, generally in image processing, the methodology is to use an algorithm accomplished by choosing a threshold automatically based on image data of histogram.

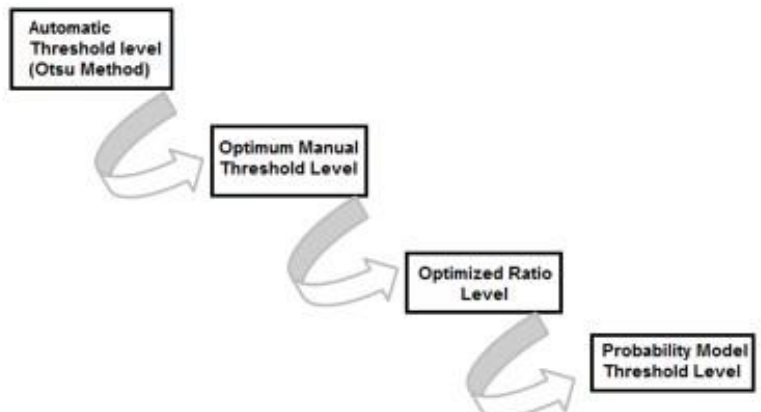

Fig. 8. Steps of Thresholding

Techniques, The subsequent iterative procedure is one such approach. The steps are followed as:

1. Select an initial estimate for the optimum manual thresholding level.

2. Segment the image using level with optimized ratio value (in which level is divided by mean value).

3. Calculate the middling intensity values for the pixels in region by probability models associated threshold value.

4. Compute a new threshold value associate in variance and standard deviation.

5. Segment the image using function im $2 \mathrm{bw}$

A. Comparisons between optimized and probability model threshold level
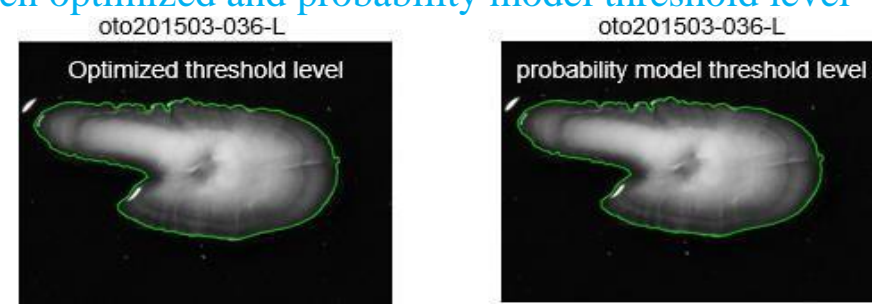

Fig. 9. Otolith image of comparison between optimized threshold level and probability model threshold level 


\section{B. Optimized threshold level}

Disadvantage: Not better contour then optimum manual threshold

Advantage: It is automatic and fast technique and better then Otsu method also less time consuming.

\section{Probability model threshold level}

Disadvantage: Not better contour then optimum manual threshold

Advantage: it is automatic, fast technique and better then Otsu method and Optimized ratio level also less time consuming.

The way to choose a threshold level by visual examination of the image histogram of optimum manual thresholding. It is mostly efficient in a collaborating position, as one allows the user to alter the threshold by a cursor associated with background and get the output straight away. The persistence of this second part was assembling additional parts of the codes and obtains an effect more precise. For this purpose, the "Otsu method" (automatic thresholding value) gray thresh code was replaced with "optimum threshold value". For succeeding the result expected, the code had to be changed again by applying the "probability model threshold" function which corrects the unvarying background illumination and manually threshold value into automatic and change the image into a binary image so it can perform analysis of the forefront objects. Performance of mean, standard deviation and variance called probability model threshold level in which we execute and result is sharp bright image with high contrast and good contouring.

\section{Region Based Segmentation and Thresholding Using MATLAB}

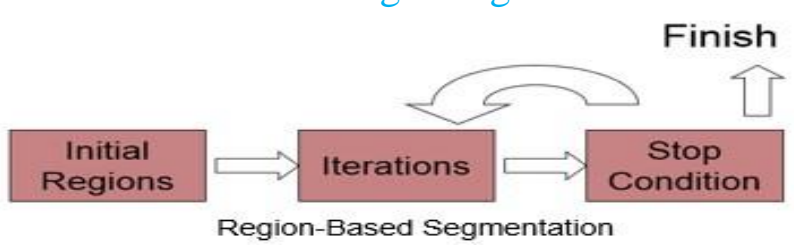

Fig. 10. Region-based segmentation of Region Growing

The purpose of segmentation is to separate an image in to the regions. In customized algorithm options were approached by this problem, by getting boundaries between regions based on discontinuities in intensity levels. The segmentation technique that is based on finding the regions is directly used here [8][12]. The scheme is to calculate seeds with level as for darker pixels the histogram is skewed right it shows high contrast intensity in an image's data set.

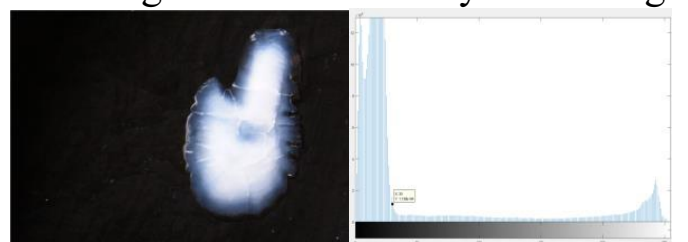

Original image "oto201411-038-L"Threshold level of "oto201411-038-L"

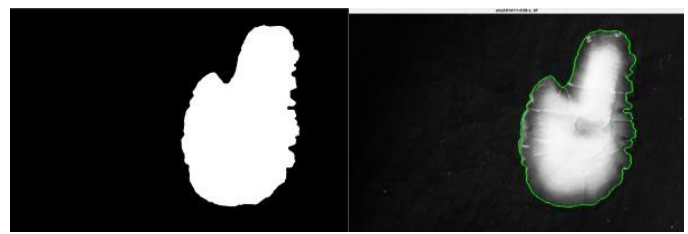

Segmented image "oto201411-038-L" Overlapped boundary green "oto201411-038-L"

Fig. 11. Region grow results

The main standard part of an algorithm is region growing based on the background of the images. And also based on the selection of level in grey threshold value while preservative connectivity if the background has sufficient homogenous that darker than outside zones of sardine otoliths. Here 
we put the value of grey threshold; I have selected those values which are corresponding with the background. These parameters may have over or under enhancement so to reduce this sharpness mean opinion score is used. [9] Final last overlapped image gives additional better results as quality of image.

E. Comparisons based Region Growing between optimum, optimized and probability model threshold level
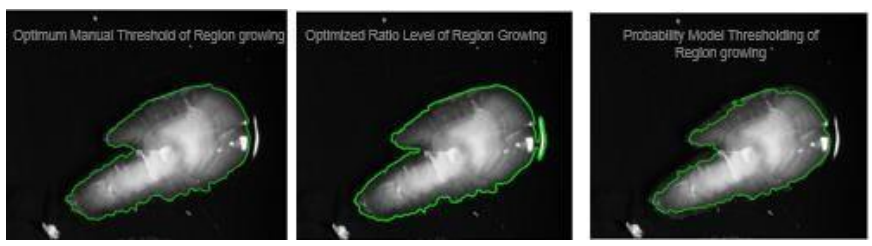

Fig. 12. oto201504-055-R of Region growing

\section{F. Evaluation of Segmentation}

The Mean Opinion Score (MOS) is stated as a particular rational number which shows quantity, usually in the assortment from 1 to 5 this process is known as "Rating scales of subjective quality Assessment". Although, 1 MOS shows lowest observed quality and 5 MOS shows the highest observed quality. The other MOS choices are also possible, it is depend on the rating (assessment) scale that has been used in the underlying test. This rating scale of absolute category is generally used, plots ratings between bad and excellent numbers among 1 and 5, as perceived in table below [10][11].

Table 2. Overlapped Of Sardine Otolith Segmentation Based Threshold Level

\begin{tabular}{|c|c|c|c|c|c|c|c|c|}
\hline S.No & $\begin{array}{l}\text { Sample } \\
\text { name }\end{array}$ & Opt1 & Opt2 & $2_{2}^{\operatorname{MOS}}$ & $\begin{array}{l}\text { Opt } \\
3\end{array}$ & $\begin{array}{l}\text { MOS } \\
3\end{array}$ & Opt 4 & $\frac{\operatorname{MOS}}{4}$ \\
\hline 1. & $\begin{array}{l}\text { oto } 20141 \\
1-038-R\end{array}$ & 105 & 24 & 4 & 24.4 & 4 & 26.8 & 4 \\
\hline 2. & $\begin{array}{l}\text { oto } 20150 \\
\text { 8-068-R }\end{array}$ & 72 & 13 & 5 & 16.7 & 4 & 19.5 & 4 \\
\hline 3. & $\begin{array}{l}\text { oto20150 } \\
7-021-L\end{array}$ & 91 & 16 & 5 & 21.1 & 4 & 22.2 & 4 \\
\hline 4. & $\begin{array}{l}\text { oto201412 } \\
-034\end{array}$ & 107 & 35 & 4 & 26.4 & 5 & 37.1 & 4 \\
\hline 5. & $\begin{array}{l}\text { oto201507 } \\
-006\end{array}$ & 99 & 17 & 4 & 24.4 & 4 & 28.8 & 4 \\
\hline
\end{tabular}

Whereas, Opt $1=$ Otsu method (automatic value), Opt 2= optimum manual threshold level, Opt $3=$ optimized threshold level, Opt 4= probability model threshold level, MOS= mean opinion score.

Table 3. Sardine Otolith Segmentation Threshold Based Region Growing

\begin{tabular}{|l|l|l|l|l|l|l|l|l|}
\hline $\begin{array}{c}\text { S. } \\
\text { No }\end{array}$ & $\begin{array}{c}\text { Sample } \\
\text { Image }\end{array}$ & Opt1 & Opt2 & $\begin{array}{c}\text { MOS } \\
2\end{array}$ & Opt3 & $\begin{array}{c}\text { MOS } \\
3\end{array}$ & Opt4 & $\begin{array}{c}\text { MOS } \\
4\end{array}$ \\
\hline 1. & $\begin{array}{c}\text { oto2014 } \\
12-014-R\end{array}$ & 81 & 14 & 5 & 18.83 & 4 & 19.9 & 4 \\
\hline 2. & $\begin{array}{c}\text { oto2014 } \\
12-033-1\end{array}$ & 109 & 28 & 4 & 25.34 & 4 & 29.8 & 4 \\
\hline 3. & $\begin{array}{c}\text { oto2014 } \\
12-033-R\end{array}$ & 112 & 37 & 4 & 26.04 & 4 & 39.04 & 4 \\
\hline 4. & $\begin{array}{c}\text { oto2014 } \\
12-035\end{array}$ & 107 & 28 & 5 & 24.87 & 5 & 53.5 & 4 \\
\hline 5. & $\begin{array}{c}\text { oto2014 } \\
12-036\end{array}$ & 123 & 38 & 4 & 28.59 & 2 & 61.5 & 4 \\
\hline
\end{tabular}

In above tables we find the quality assessment by MOS and then results are shown in bar form as seen below. These results are discussed in conclusion. 


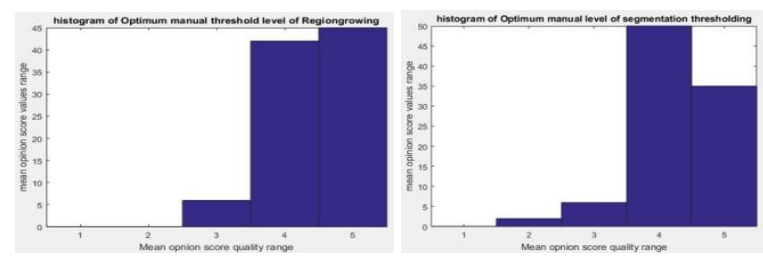

Fig. 13. Optimum manual level between segmentation of threshold technique and region growing
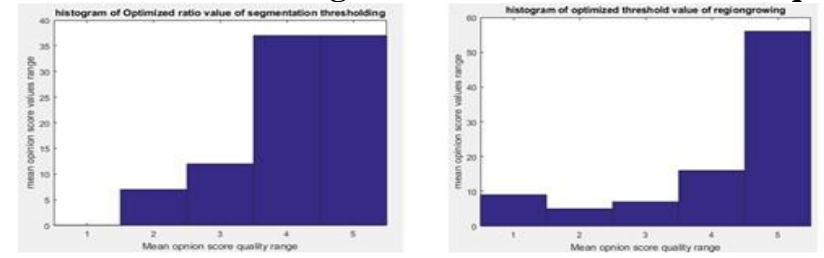

Fig. 14. Optimized Threshold Level between threshold level and region growing
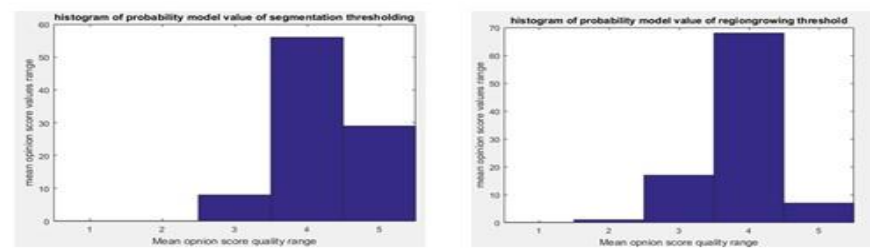

Fig. 15. Probability Model Threshold Level overlapped of sardine otolith in image segmentation based threshold level and region growing

The histograms shows contrast intensity in an image's data set, to maintain the mean brightness and to improve the contrast is necessary for image enhancement processing and algorithms.

\section{CONCLUSION}

Table 4. Comparison of All Algorithms by Data Compression

\begin{tabular}{|c|c|c|c|c|c|}
\hline & $\begin{array}{l}\text { Otsu } \\
\text { method }\end{array}$ & $\begin{array}{c}\text { Optimum } \\
\text { manual } \\
\text { level }\end{array}$ & $\begin{array}{l}\text { Optimized } \\
\text { ratio level }\end{array}$ & $\begin{array}{l}\text { Probability } \\
\text { model } \\
\text { Threshold }\end{array}$ & $\begin{array}{c}\text { Region } \\
\text { Growing }\end{array}$ \\
\hline $\begin{array}{l}\text { Advanta } \\
\text { ges }\end{array}$ & $\begin{array}{ll}\checkmark & \text { Automatic } \\
\text { Less time } \\
\text { consuming } \\
\text { Fast technique }\end{array}$ & $\checkmark \begin{array}{l}\text { Better } \\
\text { contour }\end{array}$ & $\begin{array}{ll}\checkmark & \text { Automatic } \\
\text { Less time } \\
\text { consuming } \\
r \quad \text { Fast } \\
\text { technique }\end{array}$ & $\begin{array}{ll}\text { Automatic } \\
\checkmark \text { Less time } \\
\text { consuming } \\
\text { Fast } \\
\text { technique }\end{array}$ & 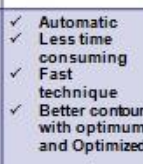 \\
\hline $\begin{array}{l}\text { Disadva } \\
\text { ntages }\end{array}$ & $\checkmark$ Bad contour & $\begin{array}{ll}\text { Non } \\
\text { automatic } \\
\text { Time } \\
\text { consuming }\end{array}$ & $\begin{array}{l}\text { Not better } \\
\text { contourt then } \\
\text { optimum } \\
\text { manual } \\
\text { threshold }\end{array}$ & $\begin{array}{l}\text { Not better } \\
\text { contour } \\
\text { then } \\
\text { optimum } \\
\text { manual } \\
\text { threshold } \\
\text { level }\end{array}$ & $\begin{array}{c}\text { Samples are } \\
\text { not good } \\
\text { contour with } \\
\text { probability }\end{array}$ \\
\hline
\end{tabular}

Better working segmentation can be done by adding morphometric of region growing method, more samples of sardine working made best to contour boundaries with this method. The intensities of the region growing histogram reveal the high contrast and sharpness of the image that is taken by manually using optimum thresholding of region growing the histogram is skewed it shows high contrast intensity in an image's data set. To maintain the mean brightness and to improve the contrast is necessary for image enhancement processing and algorithms. Good quality of image can be evaluated by contrast but it can be too sharp or too fade so mean opinion score is used to adjust quality of image for better results. This technique increases the entropy in the image so the information is getting extracted by experimenting standard data very precisely. The entropy value of the histogram is maximum and the mean intensity can also be comparable with the other histogram with mean opinion score (MOS) but it gives much better results as quality of image. Otolith segmentation with thresholding and region growing based evaluation outcomes displayed image thresholding in automatic applications. Histograms aid to resolve an appropriate thresholding method assessed by MOS that offers better outcomes for type of images in image processing. Working of projects experimental results proven that region grow segmentation method is good in thresholding images whose histogram has an extreme value related background. Many samples of otolith based 
thresholding in limited outputs with good results, but actual conditions are certainly improved reality applications, aspects to claim a predict using numerous techniques. When merging all thresholding techniques with levels applied to subcategories of the input image as mean, standard deviation and variance, results obtained with the proposed approach demonstrate better results than Otsu methods using threshold-preserving in images. For example, in images with irregular brightness, running time performance is low compared to thresholding methods when performed. On the other hand it is average in the assortment of dynamic methods, what sorts it an interesting method to try, even in realtime applications.

\section{ACKNOWLEDGEMENT}

The authors would like to thank University of Malaga and Mehran university of Engineering \& Technology Jamshoro and to all those who have supported and helped in this research study. Special thanks to the Erasmus Caprio International mobility program for providing the means to conduct these experiments in Spain.

\section{REFERENCES}

[1] E. Nava, Elisa I. Villar, María C. Clemente, Javier Rey, Alberto García, Lourdes Fernández-Peralta, Carmen G. Pineiro, and Pablo Otero. "Digital Imaging Tool to Enhance Otolith Microstructure for Estimating Age in Days in Juvenile and Adult Fish." IEEE Journal of Oceanic Engineering 43, no. 1 (2018): 48-55.

[2] B. Zorica, Zorica, Barbara, Gorenka Sinovčić, and Vanja Čikeš Keč. "Preliminary data on the study of otolith morphology of five pelagic fish species from the Adriatic Sea (Croatia)." Acta Adriatica 51, no. 1 (2010): 89.

[3] Begg, Gavin A., CRC Reef Research Centre, Mikaela AJ Bergenius, Steven X. Cadrin, and Michael F. O'Neill. Stock assessment of the Australian east coast spotted mackerel fishery. No. 58. CRC Reef Research Centre, 2005.

[4] E. Avigliano, Avigliano, Esteban, Laith A. Jawad, and Alejandra V. Volpedo. "Assessment of the morphometry of saccular otoliths as a tool to identify triplefin species (Tripterygiidae)." Journal of the Marine Biological Association of the United Kingdom 96, no. 5 (2016): 1167-1180.

[5] J. M. Long, T. B Grabowski, "Age and growth of fishes: principles and techniques", American Fisheries Society, November 2017, vol. 359.

[6] W. Schwarzhans, "A review of Jurassic and Early Cretaceous otoliths and the development of early morphological diversity in otoliths." Neues Jahrbuch für Geologie und Paläontologie-Abhandlungen 287, no. 1 (2018): 75-121.

[7] L. Shamir, J. D. Delaney, N. Orlov, D. M. Eckley and I. G. Goldberg "Pattern recognition software and techniques for biological image analysis." PLoSComputBiol, Vol. 6, No.11, 2010.

[8] N. Otsu, "A Threshold Selection Method from Gray-Level Histograms," IEEE Transactions on Systems, Man, and Cybernetics, Vol. 9, No. 1, 1979, pp. 62-https://www.udacity.com/course/computer-vision-nanodegree--nd89166.

[9] Computer Vision, https://www.udacity.com/course/computer-vision-nanodegree--nd891

[10] N. Otsu (1979). "A threshold selection method from gray-level histograms". IEEE Trans. Sys., Man., Cyber. Vol. 9 , No. 1, pp. 62-66, 1979 doi:10.1109/TSMC.1979.4310076

[11] J. Jonathan IestynMapp, MORPHOMETRIC OTOLITH ANALYSIS, University of East Anglia, School of Computing Sciences, September 2015

[12] R. C. Gonzalez, R. E. Woods, S. L. Eddins "Digital Image Processing Using Matlab”, Second edition 2009. 